\title{
Erratum to: Homoclinic orbits for an unbounded superquadratic Hamiltonian systems
}

Jun Wang, Junxiang Xu, Fubao Zhang and Lei Wang

Erratum to: Nonlinear Differ. Equ. Appl. (2010) 17:411-435 DOI 10.1007/s00030-010-0060-7

Regrettably, the last two words of the article title were omitted in the original version.

The correct article title is given below:

Homoclinic orbits for an unbounded superquadratic Hamiltonian systems

J. Wang, J. Xu, F. Zhang, L. Wang

Department of Mathematics, Southeast University,

Nanjing 210096, People's Republic of China

e-mail: wangjdn2006@21cn.com

J. Xu

e-mail: xujun@seu.edu.cn

F. Zhang

e-mail: zhangfubao@seu.edu.cn

The online version of the original article can be found under doi:10.1007/s00030-010-0060-7. 